\title{
SIMULTANEOUS APPROXIMATION AND INTERPOLATION IN $l_{1}$
}

\author{
JOSEPH M. LAMBERT
}

\begin{abstract}
In a recent paper of $\mathbf{R}$. Holmes and J. Lambert a geometrical approach was taken to the property of simultaneous approximation and interpolation which is norm preserving (SAIN), first introduced by F. Deutsch and P. Morris. An open question in both papers was if $M$ is the subspace of $l_{1}$ consisting of the elements having only finitely many nonzero components does the triple $\left(l_{1}, M, G\right)$ have property SAIN for all finite dimensional subspaces $G$ contained in $l_{\infty}$. This question is answered affirmatively by use of a generalization of Yamabe's theorem extending Helly's theorem.
\end{abstract}

0. Introduction. In [1] the concept of simultaneous approximation and interpolation which is norm preserving (SAIN) was introduced.

Definition 0.1. Let $X$ be a normed linear space, $M$ a dense subset of $X, L$ a finite dimensional subspace of $X^{*}$. The triple $(X, M, L)$ has property SAIN if for every $x \in X$ and $\varepsilon>0$ there exists $y \in M$ such that $\|x-y\|<\varepsilon,\|x\|=\|y\|$ and $\lambda(x)=\lambda(y)$ for all $\lambda \in L$.

Many examples are given in [1] of various spaces $X$, subsets $M$, and subspaces $L$ such that $(X, M, L)$ is SAIN. In particular [1, Corollary 6.2], let $M$ be the subspace of $l_{1}$ consisting of the elements having only finitely many nonzero components and let $L=\operatorname{span}\left\{y_{i}: i=1,2, \cdots, n\right\} \subset l_{\infty}$. If each $y_{i}$ is eventually constant, $\left(y_{i}=\left\{y_{i}(n)\right\}\right.$ is eventually constant if there is an index $N$ such that $y_{i}(N)=y_{i}(N+k)$ for all $\left.k\right)$ then $\left(l_{1}, M, L\right)$ is SAIN.

In [2] a geometrical approach was taken to study the concept of SAIN and in particular it was found that [2, Corollary 3] if $L$ is a finite dimensional subspace of $c_{0}$ (the predual of $\left.l_{1}\right) M$ as above, then $\left(l_{1}, M, L\right)$ is SAIN.

It was conjectured that if $L$ is any finite dimensional subspace of $l_{\infty}$ then $\left(l_{1}, M, L\right)$ is SAIN. In this paper that conjecture is verified.

In this paper the following notation and terminology is used. $X$ denotes a real normed linear space; $X^{*}$ the continuous dual of $X$, and $U(X)$ and $S(X)$ the closed unit ball and its boundary in $X$. A set $E \subset F$ is $F$ extremal, if whenever $t x+(1-t) y \in E, 0<t<1$, and $x, y \in F$ then $x, y \in E$.

Received by the editors January 22, 1971 and, in revised form, April 23, 1971.

AMS 1970 subject classifications. Primary 41A65, 41 A05.

Key words and phrases. Abstract approximation, approximation and interpolation, norm preserving approximation, Helly's theorem, Yamabe's theorem. 
An extremal subset consisting of a single point is called an extreme point. The extreme points of a set $F$ will be denoted $\operatorname{ext}(F)$. The convex hull of a set $F$ is the intersection of all convex sets containing $F$ and will be denoted $\operatorname{co}(F)$. The closure of a set $F$ will be denoted $\bar{F}$ or $\operatorname{cl}(F)$.

The real valued function $\operatorname{sgn}(\cdot)$ : Reals $\rightarrow\{-1,0,1\}$ is defined via $\operatorname{sgn}(x)=x /|x|, x \neq 0$ and $\operatorname{sgn}(0)=0$.

1. $M$-core.

Definition 1.1. Let $F$ be a set in $X, M$ a subset of $F$, then $F$ is said to have $M$-core at $f$ in $F$, if given $m$ in $M$ there exists $\delta(m, f)>0$ such that $(1+\delta) f-\delta m$ is in $F$, for $0 \leqq \delta \leqq \delta(m, f)$. Let $e_{i}=\left\{\delta_{i j}\right\}$ denote the usual set of basis vectors in $l_{1}$.

Proposition 1.1. Let $x \in S\left(l_{1}\right), x=\sum_{i \in A} x_{i} e_{i} ; x_{i} \neq 0, i \in A, A$ countable. Let $F$ be minimal closed $U\left(l_{1}\right)$ extremal subset containing $x$. Let $M=$ $\operatorname{co}\left(\operatorname{sgn}\left(x_{i}\right) e_{i}: i \in A\right)$. Then $F$ has $M$-core at $x$.

Proof. By [4, Theorem 1] every closed bounded convex subset of $l_{1}$ is the closed convex hull of its extreme points. Since the extreme points of $U\left(l_{1}\right)$ are the usual basis vectors of norm one and $F$ is $U\left(l_{1}\right)$ extremal it is easy to see that $F=\operatorname{cl}\left(\operatorname{co}\left(\operatorname{sgn}\left(x_{i}\right) e_{i}: i \in A\right)\right)$. Assume $m$ in $M$ is given. It remains to find $\delta(x, m)>0$ such that $z=(1+\delta) x-\delta m$ is in $F$. Since $m$ is in $M, m=\sum_{i \in B} m_{i} \operatorname{sgn}\left(x_{i}\right) e_{i}, B$ a finite set. It suffices to find $\delta$ such that

$$
\begin{aligned}
1=\|z\| & =\sum_{i \in A}(1+\delta) x_{i}-\delta m_{i} \\
& =(1+\delta) \sum_{i \in A / B}\left|x_{i}\right|+\sum_{i \in B}\left|(1+\delta) x_{i}-\delta m_{i}\right| .
\end{aligned}
$$

If one can insure that for $i \in B, \operatorname{sgn}\left((1+\delta) x_{i}-\delta m_{i}\right)=\operatorname{sgn}\left(x_{i}\right)$ then one would have

Thus

$$
\begin{aligned}
\sum_{i \in B}\left|(1+\delta) x_{i}-\delta m_{i}\right| & =\sum_{i \in B}\left[(1+\delta) x_{i}-\delta m_{i}\right] \operatorname{sgn}\left(x_{i}\right) \\
& =\sum_{i \in B}(1+\delta) x_{i} \operatorname{sgn}\left(x_{i}\right)-\delta \sum_{i \in B} m_{i} \operatorname{sgn}\left(x_{i}\right) \\
& =(1+\delta) \sum_{i \in B} x_{i}-\delta \sum_{i \in B} m_{i} .
\end{aligned}
$$

$$
\begin{aligned}
\|z\| & =(1+\delta) \sum_{i \in A / B}\left|x_{i}\right|+(1+\delta) \sum_{i \in B}\left|x_{i}\right|-\delta \sum_{i \in B}\left|m_{i}\right| \\
& =(1+\delta)\|x\|-\delta\|m\|=(1+\delta)-\delta=1 .
\end{aligned}
$$

This would conclude the proof. To insure $\operatorname{sgn}\left((1+\delta) x_{i}-\delta m_{i}\right)=\operatorname{sgn}\left(x_{i}\right)$ for all $i \in B$, let $\varepsilon_{i}>0$ be chosen such that $\left|x_{i}\right|>\varepsilon_{i}\left|m_{i}\right|$. Let $\delta=\min _{i \in B}\left\{\varepsilon_{i}\right\}$.

Q.E.D.

2. Yamabe's theorem. The following proposition is a version of Yamabe's theorem [1], [4] for convex sets. 
Proposition 2.1. Let $X$ be a Banach space, $F$ a convex set contained in $X$. Let $y_{1}, \cdots, y_{n} \in X^{*}$. Let $M$ be a norm dense convex subset of $F$. Let $f \in F, F$ having $M$-core at $f$; then given $\varepsilon>0$ there exists $m$ in $M$ such that $y_{i}(f)=y_{i}(m), i=1, \cdots, n$ and $\|f-m\|<\varepsilon$.

Proof. Let $K=F \cap\{b:\|b-f\| \leqq \varepsilon\}$. $K$ is closed and convex, $N=M \cap K$ is convex and dense in $K$ and $f$ is an $N$ core point for $K$. Define the continuous function $\varphi: F \rightarrow R^{n}$ via $\varphi(x)=\left(y_{1}(x), \cdots, y_{n}(x)\right)$. Then $\varphi N$ is dense in $\varphi K$ and thus $\varphi N$ contains the relative interior of $\varphi K$. Also $\varphi f$ is a $\varphi N$ core point of $\varphi K$ and this implies that $\varphi f$ is in the relative interior of $\varphi K$, hence in $\varphi N$. It follows that there is an $m \in N$ such that $\varphi m=\varphi f$.

O.E.D.

\section{Application to SAIN in $l_{1}$.}

DefinItion 3.1. Let $x \in S(X) ; F(x)$ denotes the minimal closed $U(X)$ extremal subset containing $x$.

THEOREM 3.1. Let $X$ be a Banach space, $M$ a dense subspace in $X$. Then $(X, M, L)$ is $S A I N$ for all finite dimensional subspaces $L$ in $X^{*}$, whenever $F(x) \cap M$ is dense in $F(x)$ and $F(x)$ has $F(x) \cap M$-core at $x$, for every $x \in S(X)$.

Proof. Given $x \in S(X)$, one applies Proposition 2.1 to $F(x)$ and obtains $m \in F(x) \cap M$ satisfying the SAIN conditions at $x$. The homogeneity of the functionals in $L$ and of the norm yields the result for all $x \in X$. Q.E.D.

THEOREM 3.2. Let $X=l_{1}, M$ be the subspace of vectors with only finitely many nonzero coordinates. Then for all finite dimensional subspaces $L \subset l_{\infty}$ $(X, M, L)$ is SAIN.

Proof. Given $x \in S\left(l_{1}\right) ; F(x), F(x) \cap M$ satisfy the hypotheses of Proposition 1.1. Hence, they satisfy the hypotheses of Theorem 3.1. Q.E.D.

ACKNOWLEDgement. The author wishes to thank Professor Richard Holmes of Purdue University for several helpful discussions on this topic and to the referee for the simplified proof of Proposition 2.1.

\section{REFERENCES}

1. F. Deutsch and P. Morris, On simultaneous approximation and interpolation which preserves the norm, J. Approximation Theory 2 (1969), 355-373. MR 40 \#6146.

2. R. Holmes and J. Lambert, A geometrical approach to property (SAIN) (to appear).

3. J. Lindenstrauss, On extreme points in $l_{1}$, Israel J. Math. 4 (1966), 59-61. MR 34 \#589.

4. H. Yamabe, On an extenison of the Helly's theorem, Osaka Math. J. 2 (1950), 15-17. MR 12, 616.

Department of Mathematics, Pennsylvania State University, York, PennsylVANIA 17403 